\title{
Estratégias de Comunicadores Auxiliados para Instruir Parceiros de Comunicação na Construção de Modelos Físicos ${ }^{1}$ AIDED Communicators Strategies to Instruct Communication Partners When Constructing Physical Models
}

\author{
Munique MASSARO ${ }^{2}$ \\ Kristine STADSKLEIV ${ }^{3}$ \\ Stephen von TETZCHNER ${ }^{4}$ \\ Débora DELIBERATO5
}

\begin{abstract}
RESUMO: este estudo teve como objetivo analisar as estratégias utilizadas pelos comunicadores auxiliados e comunicadores naturalmente falantes ao instruir um parceiro de comunicação para a construção de modelos físicos. Os participantes foram 18 comunicadores auxiliados e 18 comunicadores naturalmente falantes de um grupo de comparação, com idade entre 5-15 anos, do Brasil e da Noruega. Além disso, três diferentes grupos de parceiros de comunicação naturalmente falantes participaram da pesquisa: pais, colegas e profissional. Em um primeiro momento, as crianças comunicadores auxiliados foram avaliadas. Em seguida, as tarefas de construção foram administradas. Nessa tarefa, os comunicadores auxiliados e as crianças do grupo de comparação tiveram que instruir o parceiro de comunicação para construir modelos físicos. Os participantes foram filmados ao fazer a construção e as gravaçóes foram transcritas. Foi realizada a análise de conteúdo, de que resultou no delineamento de categorias, e os resultados foram analisados qualitativa e quantitativamente. Este estudo sugere que existem diferenças entre as estratégias utilizadas nas construçôes. Comunicadores auxiliados e seu parceiro de comunicaçáo usam mais tempo para concluir as construçóes. No entanto, ambos os grupos usaram a linguagem para instruir o parceiro de comunicação e finalizar as construçóes.
\end{abstract}

PALAVRAS-CHAVE: Educação Especial. Inclusão. Linguagem. Comunicação Alternativa.

ABSTRACT: This study aimed to analyze the strategies used by aided communicators and of naturally speaking communicators to instruct a communication partner for the construction of physical models. Participants were 18 aided communicators and 18 naturally speaking communicators of a comparison group, aged 5-15 years, from Brazil and Norway. In addition, three different groups of naturally speaking communication partners participated in the research: parents, peers and one professional. At first, the aided communicator children were evaluated. Next, the construction tasks were administered. In this task, aided communicators and comparison group had to instruct the communication partner to build physical models. Participants were filmed during the building and the recordings were transcribed. Content analysis was performed, which resulted in the delineation of categories, and the results were qualitatively and quantitatively analyzed. This study suggests that there are differences between the strategies used in the constructions. Aided communicators and their communication partners spent more time to complete the construction. However, both groups used the language to instruct the communication partners and finish the constructions.

KEYWORDS: Special Education. Inclusion. Language. Augmentative and Alternative Communication.

\footnotetext{
${ }^{1}$ http://dx.doi.org/10.1590/S1413-65382216000300003

Financiamento: Fundação de Amparo à Pesquisa do Estado de São Paulo, Brasil (número de concessão 2014/09050-3). Sophies Minde Foundation em Oslo e pelo Departamento de Psicologia da Universidade de Oslo, Noruega.

${ }^{2}$ Doutoranda pelo Programa de Pós-Graduação em Educação, Universidade Estadual Paulista "Júlio de Mesquita Filho", campus de Marília, São Paulo, Brasil. munique_massaro@marilia.unesp.br

3 Psicóloga na Seção de Pediatria de Neuro-habilitação, Departamento de Neurociências Clínicas para Crianças, Hospital Universitário de Oslo, Oslo, Noruega. Doutoranda pela Universidade de Oslo, Departamento de Psicologia, Oslo, Noruega. Oslo, Noruega. kristine.stadskleiv@psykologi.uio.no

4 Ph.D. e Professor do Departamento de Psicologia, Universidade de Oslo, Noruega. Oslo, Noruega. s.v.tetzchner@psykologi.uio.no

${ }^{5}$ Docente do Departamento de Educação Especial e do Programa de Pós-Graduação em Educação, Universidade Estadual Paulista "Júlio de Mesquita Filho", campus de Marília. Brasil. delibera@marilia.unesp.br
} 


\section{INTRODUÇÁo}

Na primeira infância, as crianças com desenvolvimento típico manipulam objetos que as cercam, e percebem as propriedades por suas formas, cores, tamanhos e aspectos espaciais. Isso não acontece apenas por meio da simples manipulação de objetos, mas também pela mediaçáo de um adulto. A atividade também serve de base para aprender as palavras que a criança se relaciona com os objetos e fenômenos do mundo (MUKHINA, 1996; VYGOTSKY; LURIA; LEONTIEV, 2001).

A rotina de atividades motoras compartilhadas entre os parceiros não só favorece o desenvolvimento de habilidades motoras, mas também envolve a aquisição de novos conhecimentos (COHEN, 2006; MIYAKAWA; KAMII; NAGASHIRO, 2005). A realização de uma atividade motora permite a aquisição e desenvolvimento das funções da linguagem, bem como a aquisiçáo e desenvolvimento de seus elementos, como no caso do vocabulário, sintaxe e pragmática. As crianças podem aprender sobre o mundo por meio de suas açóes e com a mediação de um parceiro que é competente na representação do sistema linguístico da sua comunidade.

Algumas crianças com deficiências motoras não têm a possibilidade de explorar ou podem ter dificuldades com a exploração do meio ambiente. Elas dependem, substancialmente, da mediação oferecida por parceiros que realizam as açóes motoras para elas. No entanto, a criança com deficiência motora grave pode observar as açóes realizadas pelo parceiro no meio ambiente. Isto implica que eles podem compartilhar os esquemas visuais e auditivos que a ação oferece, mas não as sensaçóes dos esquemas motores envolvidos (DELIBERATO; NUNES; WALTER, 2014).

O suporte ao idioma que o parceiro pode oferecer durante as experiências podem dar às crianças com deficiências motoras oportunidades para desenvolver o seu conhecimento do mundo, vocabulário e compreensão da gramática. Estas, por sua vez, poderiam tornar capazes de agir sobre o mundo por meio do seu próprio uso da linguagem. O desenvolvimento de competências linguísticas, portanto, poderia compensar a falta de habilidades motoras. Por exemplo, uma criança com limitações motoras graves pode participar de atividades lúdicas utilizando a linguagem para instruir outras pessoas para executar açóes para ela. Assim, a linguagem pode permitir que a criança participe e náo apenas observe uma atividade (BATOROWICZ; STADSKLEIV; VON TETZCHNER, 2014).

As crianças com deficiências motoras graves, muitas vezes, também têm problemas graves de fala. Sistemas de comunicação suplementar e alternativo (CSA) são utilizados para complementar ou substituir a língua falada (VON TETZCHNER, 2009). Existem diferentes formas de CSA; os mais comumente usados são sinais manuais e sinais gráficos. Se uma criança tem limitaçôes motoras graves, muito provavelmente não será possível usar sinais manuais. Crianças que usam comunicação gráfica (símbolos e ortografia) como seu principal modo de comunicação, e para quem a CSA não apenas suplementa, mas substitui o discurso, são referidas como comunicadores auxiliados. Os símbolos gráficos e as letras são apresentados em pranchas de comunicação ou em livros de comunicação. Estas placas e livros estão normalmente disponíveis tanto em papel como em dispositivos eletrônicos (VON TETZCHNER; MARTINSEN, 1996; VON TETZCHNER, 2009). 
Interações comunicativas entre as pessoas que usam a comunicação auxiliada e parceiros naturalmente falantes podem ser diferentes das pessoas que ambos são naturalmente falantes. Entre as pessoas que usam comunicação auxiliada e parceiros naturalmente falantes, estas interações podem também diferenciarem dependendo, por exemplo, das habilidades de comunicação e de linguagem de comunicadores auxiliados, do seu vocabulário disponível em um dispositivo ou placa, do meio ambiente e do estilo da conversação dos parceiros naturalmente falantes

$\mathrm{Na}$ área de CSA, há estudos que descrevem algumas características de tais interaçóes. Em geral, as pessoas que usam a comunicação auxiliada tendem a ser mais dependentes de seus parceiros. Um padrão altamente assimétrico de interação, embora síncrona e transacional, é frequente. Além disso, temas de conversas que estão principalmente relacionados com questôes imediatas, são observados (LIGHT; COLLIER; PARNES, 1985; BASIL, 1992; VON TETZCHNER; MARTINSEN, 1996; FERM; AHLSEN; BJÖRCK-ÅKESSON, 2012).

Nas interaçóes, os parceiros de comunicação expressam principalmente uma série de instruçôes, descriçôes gerais, declarações ou perguntas e usam estratégias de adivinhação com os comunicadores auxiliados. Muitas vezes, os turnos de conversa consistem em articulaçóes e repetiçôes dos sinais gráficos que os usuários de sistemas de comunicação apontam ou paráfrases de seus próprios turnos (LIGHT; COLLIER; PARNES, 1985; VON TETZCHNER; MARTINSEN, 1996; KENT-WALSH; MCNAUGHTON, 2005).

Além disso, uma parte substancial das conversas é composta pelos parceiros pedindo aos comunicadores auxiliados sobre objetos, pessoas e eventos que eles já sabem as respostas. $\mathrm{O}$ processo de troca de turno e o fluxo da conversa parece ser de importância vital para os parceiros de comunicação, assim, eles preferem usar questóes fechadas que exigem respostas simples de sim/não em oposição a questóes em aberto, o que reduz as demandas de comunicação e permite-lhes manter os turnos da conversa (LIGHT; COLLIER; PARNES, 1985).

A liderança do parceiro de comunicação nas interações pode estar associada com os juízos de valor negativos em relação a participação dos comunicadores auxiliados na interação. Os parceiros de comunicação podem não esperar uma resposta das crianças (CLARKE; KIRTON, 2003).

Apesar das estratégias dos parceiros de comunicação poderem influenciar os resultados das interaçóes comunicativas dos comunicadores auxiliados, por outro lado, os comunicadores auxiliados muitas vezes não tomam a iniciativa comunicativa e eles têm estratégias de comunicação inadequadas. Eles quase exclusivamente dão respostas específicas, expressam uma gama limitada de funçóes de comunicação, suas interações são principalmente as respostas de uma só palavra e sua comunicação é lenta (HARRIS, 1982; VON TETZCHNER; MARTINSEN, 2000; KENT-WALSH; MCNAUGHTON, 2005).

Finke e Quinn (2012) analisaram as percepçóes de fonoaudiólogos quanto ao estilo de comunicação (passivo ou ativo) de crianças que necessitam de CSA. Os autores argumentaram que as opçóes de vocabulário disponíveis para as crianças tendem a ser insuficientes para ter um estilo de comunicação ativo. Os fonoaudiólogos relataram que o vocabulário de iniciar, manter e ampliar uma interação estava disponível para todas as crianças classificadas como 
tendo o estilo de comunicação passiva ou ativa, no entanto, eles classificaram "comunicar desejos e necessidades", como o propósito comunicativo mais importante para representar em um sistema de CSA. Os autores argumentam que, ao priorizar desejos e necessidades, os fonoaudiólogos podem, sem intenção, limitar a gama de atos de comunicação das crianças.

Além disso, as pessoas que usam a comunicação auxiliada apenas controlam alguns meios para dirigir a atenção dos parceiros de comunicação em atividades, dão pistas em assuntos de situação imediata e uma grande parte dos enunciados produzidos na comunicação é mal interpretada. Comunicadores auxiliados têm problemas na reparação de mal intepretaçóes e parceiros de comunicação raramente gastam tempo reparando tais erros (VON TETZCHNER; MARTINSEN, 1996; MIDTLIN et al., 2014).

Estratégias utilizadas sem sucesso, a falta de controle do ambiente e repetidas experiências de falhas de comunicação podem diminuir a motivação para se comunicar e a crença na própria capacidade de se comunicar. Isso pode levar a impotência aprendida e pode contribuir para o papel não-dominante de muitos comunicadores auxiliados nas interaçóes, mesmo quando estes têm os recursos necessários para modificar o ambiente interacional. A impotência aprendida é o padrão de passividade encontrado em comunicadores auxiliados relacionado com a falta de capacidade de resposta dos parceiros de comunicação naturalmente falantes (BASIL, 1992).

Embora existam alguns estudos sobre o uso de estratégia na comunicação auxiliada, muito ainda não é explorado (HÖRMEYER; RENNER, 2013; BATOROWICZ; STADSKLEIV; VON TETZCHNER, 2014). De acordo com a perspectiva de que as crianças aprendem a língua por meio de interaçóes com outras pessoas e que os comunicadores auxiliados podem dominar as interaçóes comunicativas, usando uma linguagem para a ação, o presente estudo investiga as estratégias da criança nas tarefas de construção de modelos físicos. Nestas tarefas, os comunicadores auxiliados tiveram que instruir o parceiro de comunicação naturalmente falante para construir modelos físicos parecidos com modelos originais. As estratégias que os comunicadores auxiliados utilizaram foram comparadas aos das crianças naturalmente falantes.

\section{Objetivo}

Analisar as estratégias utilizadas pelos comunicadores auxiliados e crianças falantes naturais ao instruir um parceiro de comunicação para a construção de modelos físicos.

\section{Método}

O estudo é parte de um projeto internacional que explora conquistas e desafios de comunicadores auxiliados e foi aprovado pelos comitês de ética adequados em cada país (VON TETZCHNER et al., 2013).

\subsection{Participantes}

Os participantes foram 18 comunicadores auxiliados e 18 crianças naturalmente falantes de um grupo de comparaçáo, com idade entre cinco e 15 anos, do Brasil e da Noruega. 
Os critérios de seleção dos comunicadores auxiliados foram: a) idade entre cinco e 15 anos; b) audição e visão normal (com tecnologia corretiva); c) compreensão da fala considerado adequado ou próximo do adequado para a idade; d) falta de produção de fala ou fala muito difícil de entender; e) ter usado alguma forma de auxílio à comunicação por mais de 12 meses (auxílio manual ou eletrônico); f) não considerado com deficiência intelectual por seus professores; g) não ter diagnóstico do espectro do autismo.

As crianças do grupo de comparação eram naturalmente falantes do mesmo sexo dos comunicadores auxiliados. Elas eram da mesma classe ou de uma escola nas proximidades; se o comunicador auxiliado frequentava uma escola especial, foi selecionada uma criança mais próxima em idade do comunicador auxiliado. As crianças do grupo de comparação eram saudáveis, não tinha deficiências sensoriais conhecidos ou dificuldades de aprendizagem.

A idade média de ambos os grupos foi de 11,6 anos (variação 6;0 - 15;10). Não houve diferença significativa na idade entre os comunicadores auxiliados e crianças naturalmente falantes $(\mathrm{M}=137,9$ vs 138,1 meses, $\mathrm{t}=-0,018, \mathrm{p}=0,986)$.

Tanto os comunicadores auxiliados como os comunicadores do grupo de comparação concluíram as tarefas em conjunto com parceiros de comunicação que eram naturalmente falantes. Havia três diferentes grupos de parceiros de comunicação: pais, colegas e profissionais. Todos os parceiros de comunicação estavam familiarizados com a criança e com a comunicação auxiliada. Todos os participantes residentes no Brasil eram falantes nativos do português e todos os participantes da Noruega eram falantes nativos do norueguês.

A maioria dos comunicadores auxiliados usava cadeira de rodas para a mobilidade. Quatorze deles foram classificados no nível IV e V, e quatro no nível I e II do Gross Motor Function Classification System (GMFCS) (PALISANO et al., 1997). Além disso, a maioria tinha capacidade limitada para lidar com objetos e realizar outras ações motoras usando as mãos. Treze foram classificados no nível IV e V, e cinco sobre o nível I, II e III do Manual Ability Classification System (MACS) (ELIASSON et al., 2006). Todos os comunicadores naturalmente falantes estavam no nível I em ambas as medidas. GMFCS e MACS têm cinco níveis, com nível I, indicando melhor possível funcionamento e nível $\mathrm{V}$ dificuldades graves. No nível I de GMFCS, a criança anda sem ajuda, nível $V$ significa que a criança não pode se sentar ou ficar de forma independente. No nível I de MACS, a criança manipula objetos facilmente e com sucesso; no nível $\mathrm{V}$ a criança não é capaz de manipular objetos.

Communication Functioning Classification System (CFC) (HIDECKER et al., 2011) é uma medida da função comunicativa cotidiana (independente da modalidade), com nível I indicando melhor funcionamento e nível $\mathrm{V}$ a comunicação mais severamente afetada. No nível I a criança pode se comunicar de forma eficiente e sem velocidade reduzida com parceiros conhecidos e desconhecidos, enquanto em nível $\mathrm{V}$ a criança tem dificuldades de ser compreendida por parceiros familiares. Sete comunicadores auxiliados foram classificados no nível IV, quatro no nível III, e sete no nível II. Todos os comunicadores naturalmente falantes estavam no nível I. 


\subsection{Procedimentos}

Os dados foram coletados de 2011 a 2014, no Brasil e na Noruega, em três lugares diferentes: na Universidade; nas casas dos participantes, e também em suas escolas. Primeiro, os participantes foram selecionados, avaliados e, em seguida, as tarefas de comunicação foram administradas. Uma dessas tarefas de comunicação era uma construção de modelos físicos.

Nessa tarefa de construçáo, comunicadores auxiliados e crianças naturalmente falantes do grupo de comparaçáo tiveram que instruir o parceiro de comunicação naturalmente falante para construir modelos físicos (carregamento do caminhão, vestir uma boneca, fazer uma série de formas geométricas, construção de uma torre de lego e estabelecer um padrão de dominó), de modo semelhante aos modelos originais. Havia duas construçóes de cada tipo, sendo um total de 10 construçóes. Os modelos originais eram vistos apenas pela criança (o grupo de comunicação auxiliada e comparaçáo), de modo que o parceiro (pai, colega ou profissional) tinha que receber as instruçóes dadas pela criança, a fim de construir os modelos físicos. As crianças podiam ver os objetos e o processo de construção do parceiro e depois adaptar a sua comunicação com a situação.

As tarefas de construção foram projetadas para que o comunicador auxiliado tivesse responsabilidade e autonomia, pois era só ele ou ela que podia ver os modelos originais. Em cada construção, as crianças deviam identificar e descrever os objetos que variavam em dimensões como cor, tamanho, número e forma. Todos os objetos foram selecionados de modo a serem fáceis de reconhecer (objetos de jogo comum) e de modo que tivessem as mesmas funções, independentemente da cultura. Além de descrever os objetos, a criança também devia descrever como o parceiro tinha que construir o modelo físico; por exemplo, um bloco de Lego para começar e em seguida onde colocar o bloco seguinte em relação ao primeiro.

A criança e as interaçôes com o parceiro foram gravadas em vídeo com duas câmeras e as interaçóes comunicativas foram transcritas, seguindo o sistema de notaçáo proposta por von Tetzchner e Basil (2011). As interações do Brasil foram transcritas em português e traduzidas para o inglês; e as interaçóes da Noruega foram transcritas em norueguês e também traduzidas para o inglês.

Em seguida, realizou-se a análise de conteúdo, de que resultou no delineamento de categorias e os resultados foram analisados de forma qualitativa e quantitativa, utilizando amostras independentes t-testes.

\subsection{ANÁLISE}

O tempo gasto para executar a construção (duas construçôes em um conjunto de construção), e as estratégias de instrução dos grupos de comunicadores auxiliados e naturalmente faltantes com seus parceiros de comunicação foram comparadas.

As estratégias foram analisadas em termos da quantidade de vocabulário utilizado, das confirmaçôes e negaçóes que as crianças forneceram e se ou não as crianças apontaram para um objeto. Também foi analisado o turno de enunciado em que a criança forneceu. Foi considerado como turno de enunciado todas expressóes, desde palavras isoladas às frases completas. As categorias de análise são apresentadas no Quadro 1. 


\begin{tabular}{|l|l|}
\hline \multirow{4}{*}{ Estratégias da criança } & Apontar para objetos \\
\cline { 2 - 2 } & Vocabulário \\
\cline { 2 - 2 } & Dar confirmação \\
\cline { 2 - 2 } & Negar escolha ou enunciado do parceiro \\
\hline
\end{tabular}

Quadro 1 - Categorias de análise.

Fonte: elaboraçấo própria.

As categorias estabelecidas no Quadro 1 foram:

- Apontar para objetos: quando a criança aponta para qualquer objeto do ambiente durante a instrução para a construção de modelos físicos.

- Vocabulário: todo tipo de palavra utilizada para instruir o parceiro na construção de modelos físicos.

- Dar confirmação: declarações de confirmação de escolha ou enunciado do parceiro, como "sim", "m-hm", (balançando a cabeça).

- Negar escolha ou enunciado do parceiro: declaraçóes de negação de escolha ou enunciado do parceiro, como "não", "eh-eh", (balançando a cabeça).

\section{Resultados E Discussó́ES}

$\mathrm{Na}$ seção de resultados e discussóes serão apresentados os resultados quantitativos e qualitativos e as estratégias das crianças. Nos exemplos será usada a legenda: $\mathrm{A}=$ comunicador auxiliado; $\mathrm{PA}$ = parceiro de comunicação do comunicador auxiliado; $\mathrm{C}=$ criança do grupo de comparaçáo; e PC = parceiro de comunicação da criança do grupo de comparação.

Tabela 1 - Comparação das construçóes do grupo de comunicação auxiliada (CA) e grupo de comparação

\begin{tabular}{l|l|l|l|l}
\hline & $\begin{array}{l}\text { Grupo CA } \\
\text { Média }(D P)\end{array}$ & $\begin{array}{l}\text { Grupo de compa- } \\
\text { raçáo } \\
\text { Média }(D P)\end{array}$ & t & p \\
\hline Tempo médio por construção & $414.1(255.8)$ & $85.41(48.8)$ & 5.357 & $.000^{* *}$ \\
\hline Tempo médio da primeira construção & $437.5(298.3)$ & $85.4(41.4)$ & 4.954 & $.000^{* *}$ \\
\hline Tempo médio da segunda construção & $378.5(246.8)$ & $84.3(55.3)$ & 4.921 & $.000^{* *}$ \\
\hline Turno de enunciado & $29.9(20.5)$ & $7.2(3.1)$ & 4.652 & $.000^{* *}$ \\
\hline Vocabulário & $13.6(9.1)$ & $43.9(20.9)$ & -5.620 & $.000^{* *}$ \\
\hline Apontar para objetos & $2.1(4.2)$ & $0.3(0.5)$ & 1.749 & .098 \\
\hline Dar confirmaçáo & $10.5(9.1)$ & $1.8(1.7)$ & 4.019 & $.001^{* *}$ \\
\hline Negar escolha ou enunciado do parceiro & $4.5(3.9)$ & $0.9(1.1)$ & 3.770 & $.001^{* *}$ \\
\hline
\end{tabular}

Fonte: elaboração própria.

Amostras independentes de t-test, variâncias iguais não assumida

${ }^{*} \mathrm{p}<.05$

** $\mathrm{p}<.01$

As duplas de comunicadores auxiliados usaram significativamente mais tempo do que as duplas do grupo de comparação (414,1 vs. 85,1 segundos). Ambos os grupos utilizaram 
mais tempo na primeira tarefa $(437,5$ e 85,4$)$ do que na segunda $(378,5$ e 84,3$)$, o que indica que houve um efeito de aprendizagem a partir do primeiro para o segundo elemento do mesmo tipo de construção (como vestir a boneca no primeiro e no segundo tempo). A diferença entre o primeiro e o segundo item não foi significativa para as duplas de comunicadores auxiliados $(\mathrm{t}$ [17 df] $=1,497, \mathrm{p}=0,153$, amostras pareadas de t-teste) ou o grupo de comparação ( $\mathrm{t}$ [15 df] $=0,165, \mathrm{p}=.871)$. No total foram 135 tarefas analisadas: 68 tarefas na primeira construção e 67 tarefas na segunda construção.

Tanto os comunicadores auxiliados como os comunicadores naturalmente falantes usaram a linguagem para instruir o parceiro de comunicação e resolver as construçóes. Eles produziram as declaraçóes que foram necessárias para o parceiro de comunicação construir os modelos físicos.

Entretanto, há uma diferença significativa entre os grupos em relação ao turno de enunciado e quantidade de vocabulário utilizado (ver Tabela 1). Isso pode ser devido a comunicadores auxiliados usarem mais vezes apenas uma ou poucas palavras em cada enunciado, exigindo, portanto, mais turnos para descrever um objeto. Crianças do grupo de comparação descreveram o objeto mais abrangente em um primeiro momento e muitos dos turnos entre eles e seus parceiros foram utilizados apenas para confirmar ou negar uma escolha feita pelo parceiro. Um exemplo disso pode ser encontrado nos exemplos 1 e 2 . O exemplo 1 é um extrato de uma conversa entre o comunicador auxiliado utilizando símbolos gráficos e o parceiro de comunicação.

(1) A: Esta BLUSA

PA: uma blusa

A: CAMISETA (aponta para a cor verde do símbolo camiseta)

PA: Verde?

A: "yes" (balançando a cabeça)

PA: Então, este é aqui, ok? (aponta para a jaqueta verde)

A: "yes” (balançando a cabeça)

PA: (veste a jaqueta verde no boneco)

O exemplo 2 é um extrato de uma conversa entre a criança do grupo de comparação e o seu parceiro de comunicação.

C: Uma jaqueta verde com capuz marrom

PC: (veste a jaqueta verde no boneco)

Comparando comunicadores auxiliados e naturalmente falantes, os comunicadores auxiliados confirmaram e rejeitaram as escolhas e declaraçóes dos parceiros significativamente mais vezes. No geral, o grupo auxiliado usou confirmaçóes significativamente mais frequentemente do que negaçóes $(\mathrm{M}(\mathrm{DP})=10,5(9,1)$ vs 4,5 $(3,9)$, t $(17 \mathrm{df})=3,733 \mathrm{p}=.002$, amostras 
pareadas t-test). Isto está de acordo com os resultados de pesquisas anteriores (HÖRMEYER; RENNER, 2013).

Os exemplos 3 e 4 mostram alguns aspectos do uso do vocabulário por comunicadores auxiliados e naturalmente falantes. Ambos nem sempre utilizaram o vocabulário preciso para instruir o parceiro de comunicação na tarefa de construção. No exemplo apresentado, os dois grupos usaram a palavra "redondo" para significar "cilindro". Isso pode ter ocorrido, no entanto, por diferentes razóes.

No exemplo mostrado, o comunicador auxiliado não poderia utilizar o símbolo gráfico para o cilindro, pois este símbolo não foi representado em sua prancha de comunicação. Não se pode saber precisamente o motivo pela qual a criança naturalmente falante não disse cilindro, no entanto, é plausível que ela tenha esquecido ou ainda náo tenha aprendido o nome desta forma. A última explicação parece mais improvável, pois essa criança naturalmente falante tem 14 anos de idade e, portanto, deveria ter aprendido isso na escola. O exemplo 3 é um extrato de uma conversa entre o comunicador auxiliado utilizando símbolos gráficos e o parceiro de comunicação.

(3) A: \{\}$B I G$ este (aponta para o quadrado vermelho do símbolo $B I G$ ) (gestos circulares sobre os símbolos FAT) $B I G$ (aponta para o quadrado vermelho do símbolo $B I G$ )

PA: Um quadrado

A: (gestos circulares sobre os símbolos FAT)

PA: Amarelo?

R: Não BIG (aponta para o quadrado vermelho do símbolo BIG) (gestos circulares sobre os símbolos FAT)

PA: A cor é vermelho?

A: $E^{\prime}$

PA: Hum? Ah, ok. Este aqui (mostra o cilindro vermelho). Gordinho, redondo e vermelho.

A: "yes” (balançando a cabeça)

PA: (risos) Coloca na cordão?

A: "yes” (balançando a cabeça)

PA: (coloca o cilindro vermelho no cordão)

O exemplo 4 é um extrato de uma conversa entre a criança do grupo de comparação e seu parceiro de comunicação.

(4) C: E um, este redondo vermelho (aponta para a mesa)

PC: (coloca o cilindro vermelho no cordão)

Comunicadores auxiliados que usam símbolos gráficos sempre terá um vocabulário expressivo limitado. Devido a isso, a qualidade das opçóes de vocabulário disponíveis para eles é fundamental e decisiva para a sua capacidade de expressar significados variados e comple- 
xos (VON TETZCHNER; MARTINSEN, 2000; BREKKE; VON TETZCHNER, 2003; FINKE; QUINN, 2012).

Em todos os exemplos acima 1-4, pode-se também observar as diferenças entre os grupos nas interaçóes entre as crianças e os parceiros. Na díade de comunicador naturalmente falante e seu parceiro de comunicação, o parceiro apenas executou uma ação e não pronunciou nada. Comunicador auxiliado e seu parceiro de comunicação teve uma interação, e um co-construção de significados do vocabulário utilizado. Às vezes, como pode ser visto no exemplo 3, o comunicador auxiliado usou uma estratégia em que ele combinou o uso de símbolos gráficos e gestos para expressar-se e o parceiro de comunicação teve que co-produzir, interpretar e traduzir o significado do enunciado, a fim de completar a tarefa. Durante a solução da tarefa, o parceiro de comunicação entendeu as estratégias utilizadas pelo comunicador auxiliado (HÖRMEYER; RENNER, 2013; BREKKE; VON TETZCHNER, 2003).

As crianças comunicadoras auxiliadas apontaram para objetos durante a conversa com mais freqüência do que o grupo de comparação, mas a diferença não foi estatisticamente significativa (ver Tabela 1). Apontar um objeto para complementar os enunciados do comunicador auxiliado pode ser parte de uma estratégia de comunicação auxiliada e economizar tempo para selecionar um item (VON TETZCHNER; MARTINSEN, 1996). No entanto, o uso do apontar para objetos que substituem o uso de expressóes com suportes de comunicação, pode refletir as limitadas possibilidades expressivas de comunicadores auxiliados. Quando as crianças se comunicam com gestos ou apontando para objetos, o parceiro de comunicação tem mais responsabilidade na conversa e precisa expressar, interpretar, traduzir e confirmar mensagem das crianças, bem como formular e expressar sua própria mensagem (HARRIS, 1982).

Comunicadores auxiliados que usaram o apontar durante a construção de modelos físicos podem não ter vocabulário disponível em sua prancha de comunicação para se expressarem, podem não usar a pasta de comunicação com o seu parceiro específico, ou podem ter estratégias de comunicação inadequadas. Esses comunicadores auxiliados utilizam um padrão de interação, principalmente relacionada com a emissão imediata.

Os resultados indicam que as crianças naturalmente falantes e seus parceiros de comunicação são mais independentes, descreveram um objeto com detalhes, principalmente em um único enunciado, exigindo menos tomadas de turno e menos tempo para completar as tarefas; conseqüentemente, houve menor interação para díade. Comunicadores auxiliados e seus parceiros de comunicação são mais dependentes, houve interação para díade e uma co-construção de significados na construção de modelos físicos.

O estudo da Ferm, Ahlsen e Björck-Åkesson (2012) discutiu que a criança naturalmente falante e seu cuidador eram menos dependentes no feedback explícito e na interpretação dentro da interação durante uma atividade de refeição e eram menos dependentes de mudanças nas contribuiçôes, a fim de cumprir os objetivos práticos das refeições. Estes resultados estão de acordo com os resultados encontrados durante atividades para a construção de modelos físicos. 


\section{Conclusão}

Os resultados mostraram que há diferenças entre as estratégias utilizadas pelos comunicadores auxiliados e naturalmente falantes. Ambos os grupos usaram a linguagem para instruir o parceiro de comunicação e resolver as construçóes. Entretanto, esta pesquisa indica que comunicadores auxiliados têm limitada experiência de usar a linguagem para este propósito.

Os comunicadores auxiliados são capazes de usar a linguagem para a ação, tornando-se um agente humano. Eles podem participar ativamente das práticas sociais diversas, em situaçóes da vida real, em conversas onde as crianças têm verdadeira responsabilidade comunicativa e podem dar sentido à vida. Além disso, a linguagem utilizada por elas pode compensar a falta de habilidades motoras que são necessárias para atuarem diretamente sobre o mundo físico.

Essas tarefas têm mostrado que é possível para as crianças, que usam comunicação auxiliada, liderar conversas, instruir outras pessoas e tomar a iniciativa nas interaçóes. As intervenções dirigidas a parceiros de comunicação são fundamentais para o processo de desenvolvimento da linguagem dos comunicadores auxiliados. Os parceiros de comunicação têm um papel decisivo no uso de estratégias de linguagem co-construídas centrados na criança durante as interaçóes com os comunicadores auxiliados e no suporte na comunicação suplementar e alternativa dada.

Não existem mais estudos sobre as estratégias de instrução utilizadas pelos comunicadores auxiliados e seus parceiros de comunicação resolvendo construção de modelos físicos, assim, futuras pesquisas poderiam ser utilmente dirigidas para analisar de forma mais clara as estratégias de instrução. O presente estudo é limitado pela pequena amostra analisada e os resultados terão de ser confirmados em estudos futuros.

\section{REFERÊNCIAS}

BATOROWICZ, B.; STADSKLEIV, K.; VON TETZCHNER, S. Participation of aided communicators in play with peers: language for action. In: BIENNAL CONFERENCE OF THE INTERNATIONAL SOCIETY FOR AUGMENTATIVE AND ALTERNATIVE COMMUNICATION, 16., 2014, Lisboa. Proceedings of $16^{\text {th }}$ Biennal Conference of the International Society for Augmentative and Alternative Communication. Toronto: ISAAC, 2014. p.1-3.

BASIL, C. Social interaction and learned helplessness in severely disabled children. Augmentative and Alternative Communication, Toronto, v.8, p.188-99, 1992.

BREKKE, K. M.; VON TETZCHNER, S. Co-construction in graphic language development. In: VON TETZCHNER, S.; GROVE, N. (Org.). Augmentative and Alternative Communication: developmental issues. London: Whurr Publishers, 2003. p.176-210.

CLARKE, M.T.; KIRTON, A. Patterns of interaction between children with physical disabilities using augmentative and alternative communication systems and their peers. Child Language Teaching and Therapy, Sheffield, v.19, p.135-51, 2003.

COHEN, L. Young children's discourse strategies during pretend block play: a sociocultural approach. 2006. 201f. Thesis (Ph.D.), Fordham University, New York, 2006. 
DELIBERATO, D; NUNES, L.R.P.; WALTER, C.C.F. Linguagem e Comunicação alternativa: caminhos para a interação e comunicação. In: ALMEIDA, M.A.; MENDES, E.G. (Org.). A escola e o público-alvo da educaçâo especial: apontamentos atuais. São Carlos: Marquezine \& Manzini; Marília: ABPEE, 2014. p.197-210.

ELIASSON, A.C. et al. The Manual Ability Classification System (MACS) for children with cerebral palsy: scale development and evidence of validity and reliability. Developmental Medicine \& Child Neurology, Malden, v.48, p.549-54, 2006.

FERM, U.; AHLSÉN, E.; BJÖRCK-AKESSON, E. Patterns of communicative interaction between a child with severe speech and physical impairments and her caregiver during a mealtime activity. Journal of Intellectual \& Developmental Disability, Newcastle, v.37, p.11-26, 2012.

FINKE, E.H.; QUINN, E. Perceptions of communication style and influences on intervention practices for young children with AAC needs. Augmentative and Alternative Communication, Toronto, v.28, p.117-26, 2012.

HARRIS, D. Communicative interaction processes involving nonvocal physically handicapped children. Topics in language disorders, Michigan, v.2, p.21-37, 1982.

HIDECKER, M.J.C. et al. Developing and validating the Communication Function Classification System (CFCS) for Individuals with Cerebral Palsy. Developmental Medicine and Child Neurology, Malden, v.53, p.704-10, 2011.

HÖRMEYER, I.; RENNER, G. Confirming and denying in co-construction processes: A case study of an adult with cerebral palsy and two familiar partners. Augmentative and Alternative Communication, Toronto, v.29, p.259-71, 2013.

KENT-WALSH, J.; MCNAUGHTON, D. Communication Partner Instruction in AAC: present practices and future directions. Augmentative and Alternative Communication, Toronto, v. 21, p.195204, 2005.

LIGHT, L.; COLLIER, B.; PARNES, P. Communicative Interaction between Young Nonspeaking Physically Disabled Children and Their Primary Caregivers: Part 1 - Discourse Patterns. Augmentative and Alternative Communication, Toronto, v.4, p.74-83, 1985.

MIDTLIN, H.S. et al. What communication strategies do AAC users want their communication partners to use? A preliminary study. Disability and Rehabilitation, Ipswich, v.22, p.1-8, 2014.

MIYAKAWA, Y.; KAMII, C.; NAGASHIRO, M. The development of logico mathematical thinking at ages 1-3 in play with blocks and an incline. Journal of Research in Child Development, Malden, v. 19, p.292-301, 2005.

MUKHINA, V. Psicologia da idade pré-escolar. São Paulo: Martins Fontes, 1996.

PALISANO, R. et al. Development and reliability of a system to classify gross motor function in children with cerebral palsy. Developmental Medicine and Child Neurology, Malden, v.39, p.214-23, 1997.

VYGOTSKY, L.S.; LURIA, A.R.; LEONTIEV, A.N. Linguagem, desenvolvimento e aprendizagem. São Paulo: Icone, 2001.

VON TETZCHNER, S. Suporte ao desenvolvimento da comunicacao suplementar e alternativa. In: DELIBERATO, D.; GONÇALVES, M.J.; MACEDO, E.C. (Org.). Comunicação alternativa: teoria, prática, tecnologias e pesquisa. São Paulo: Memnon Ediçóes Cientificas, 2009. p.14-27. 
VON TETZCHNER, S. et al. Becoming aided communicators: Communicative development and problem solving in children using communication aids. Book in preparation. 2013.

VON TETZCHNER, S.; BASIL, C. Terminology and notation in written representations of conversations with augmentative and alternative communication. Augmentative and Alternative Communication, Toronto, v.27, p. 141-149, 2011.

VON TETZCHNER, S.; MARTINSEN, H. Words and strategies: conversations with young children who use aided language. In: VON TETZCHNER, S.; JENSEN, M.H. (Org.). Augmentative and alternative communication: European Perspectives. London: Whurr Publishers Ltd, 1996. p. 65-88.

VON TETZCHNER, S.; MARTINSEN, H. Introdução à comunicação aumentativa e alternativa. Porto: Porto Editora, 2000.

Recebido em: 15/06/2015

Reformulado em: 23/05/2016

Aprovado em: 30/05/2016 
MASSARO, M. et al. 